\title{
DNA methylation analysis of negative pressure therapy effect in diabetic foot ulcers
}

\author{
A H Ludwig-Slomczynska' ${ }^{1}$, S Borys ${ }^{2,3}$, M T Seweryn ${ }^{1}$, J Hohendorff',3, P Kapusta' ${ }^{1}$, B Kiec-Wilk ${ }^{2,3}$, E Pitera', \\ P P Wolkow ${ }^{1}$ and M T Malecki ${ }^{2,3}$ \\ ${ }^{1}$ Center for Medical Genomics OMICRON, Jagiellonian University Medical College, Krakow, Poland \\ ${ }^{2}$ Department of Metabolic Diseases, Jagiellonian University Medical College, Krakow, Poland \\ ${ }^{3}$ University Hospital, Krakow, Poland
}

Correspondence should be addressed to P Wolkow or M T Malecki: pawel.wolkow@uj.edu.pl or maciej.malecki@uj.edu.pI

\begin{abstract}
Objective: Negative pressure wound therapy (NPWT) has been used to treat diabetic foot ulcerations (DFUs). Its action on the molecular level, however, is only partially understood. Some earlier data suggested NPWT may be mediated through modification of local gene expression. As methylation is a key epigenetic regulatory mechanism of gene expression, we assessed the effect of NPWT on its profile in patients with type 2 diabetes (T2DM) and neuropathic non-infected DFUs.

Methods: Of 36 included patients, 23 were assigned to NPWT and 13 to standard therapy. Due to ethical concerns, the assignment was non-randomized and based on wound characteristics. Tissue samples were obtained before and $8 \pm 1$ days after therapy initiation. DNA methylation patterns were checked by Illumina Methylation EPIC kit. Results: In terms of clinical characteristics, the groups presented typical features of T2DM; however, the NPWT group had significantly greater wound area: $16.8 \mathrm{~cm}^{2}$ vs $1.4 \mathrm{~cm}^{2}(P=0.0003)$. Initially only one region at chromosome 5 was differentially methylated. After treatment, 57 differentially methylated genes were found, mainly located on chromosomes 6 (chr6p21) and 20 (chr20p13); they were associated with DNA repair and autocrine signaling via retinoic acid receptor. We performed differential analyses pre treatment and post treatment. The analysis revealed 426 differentially methylated regions in the NPWT group, but none in the control group. The enrichment analysis showed 11 processes significantly associated with NPWT, of which 4 were linked with complement system activation. All but one were hypermethylated after NPWT. Conclusion: The NPWT effect on DFUs may be mediated through epigenetic changes resulting in the inhibition of complement system activation.
\end{abstract}

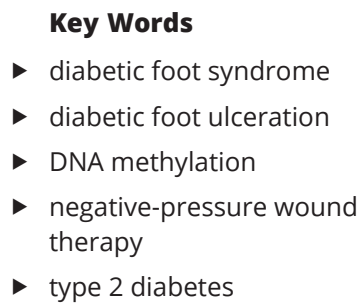

\section{Key Words}

diabetic foot syndrome

- DNA methylation

negative-pressure wound

type 2 diabetes

\section{Introduction}

Diabetic foot syndrome (DFS) is characterized as an infection, ulceration, or destruction of deep tissues of the foot associated with neuropathy and/or peripheral arterial disease (1). Diabetic foot ulcer (DFU) is a frequently diagnosed form of DFS. The yearly incidence rate reported among patients with diabetes has reached $6.0 \%$ (2). It is associated with a high rate of recurrence, amputation, and mortality (3). The standard management of the DFU includes glycemic control, offloading, revascularization, antibiotics, surgical debridement and different topical dressings. Despite the new treatment modalities for DFUs, many patients still suffer from prolonged treatment courses and concomitant complications. There is subsequently a significant demand for adjunct therapies to improve the prognosis of these patients. Among supportive strategies, negative pressure wound therapy (NPWT) is emerging as 
an area of a great clinical interest and research. It has found its application in many clinical situations, including DFU management $(4,5)$. NPWT is based on the use of occlusive dressings applied to the wound with subatmospheric pressure gradients, typically $-75 \mathrm{mmHg}$ to $-125 \mathrm{mmHg}$, usually continuous, transmitted through drains from a vacuum generator. NPWT causes tissue deformations facilitating wound borders closure. It stimulates a wound healing cascade that includes exudate removal, reduction of edema, neoangiogenesis, and granulation promotion (reviewed in (6)). Its mechanism of action on the molecular level, however, is only partially understood. NPWT results in a local tissue alteration toward more proangiogenic and anti-inflammatory molecular conditions. Specifically, growth factors, inflammatory cytokines, and matrix metalloproteinases constitute molecular pathways affected by this therapy (reviewed in (6)). Previous animal and human data suggested that it might act through modification of local gene expression $(7,8)$; however, most of them focused on particular molecular pathways and the analysis was performed by Q-PCR methods. Recently, we conducted an analysis in which we characterized transcriptome wide gene expression profile using Illumina microarrays in patients with type 2 diabetes (T2DM) and DFU in comparison with standard therapy (9). In light of this prior evidence of differential expression of several genes between the two treatment methods (9), it seemed strongly justified to test whether these can also be seen at the epigenetic level of gene methylation, a key regulatory mechanism of gene expression.

In this study, we performed a genome-wide DNA analysis to compare the effect of NPWT versus standard therapy on the methylation profile in the wound bed of patients with type 2 diabetes (T2DM) and neuropathic, non-infected DFUs.

\section{Methods}

\section{Patients}

Thirty-six consecutive patients with T2DM and DFUs from an outpatient clinic of the Department of Metabolic Diseases, University Hospital, Krakow, a tertiary academic outpatient center for patients with DFS in southern Poland, were selected using previously described criteria (9). Patients were qualified to the study if they had peripheral diabetic polyneuropathy (diagnosed with at least two standard clinical tests) complicated by superficial neuropathic foot ulcerations. We included patients with the presence of no more than three neuropathic, non-infected, non-ischemic ipsilateral foot wounds. Specific inclusion criteria comprised (a) a clinical diagnosis of T2DM and (b) the presence of no more than three neuropathic, clinically non-infected foot wounds. Exclusion criteria included (a) clinically significant ischemia defined by the lack of pulses of both main pedal arteries and/or an ankle-brachial index less than 0.9, (b) symptoms of infection, (c) bilateral ulcerations, (d) active osteomyelitis, and (e) active Charcot foot (based on clinical examination and X-ray imaging). The patients were assigned either to standard therapy alone $(n=13)$ or standard combined with NPWT $(n=23)$. The assignment to NWPT was non-random and based on wound characteristics, mainly area $\left(>1.0 \mathrm{~cm}^{2}\right)$. This was done to mirror the current use of NPWT in patients with larger foot wounds in routine clinical practice (10, $11,12)$. Patients with smaller ulcerations $\left(\leq 1.0 \mathrm{~cm}^{2}\right)$ were assigned to the comparator group. However, in the presence of technical difficulties for NPWT application (e.g. unfavorable localization for placement) or lack of patient consent for NPWT, patients were allocated to the comparator group as well. A commercial NPWT system (Renasys EZ Plus, Smith \& Nephew, London, UK) was used and continuous negative pressure of $-120 \mathrm{mmHg}$ was applied to the ulceration filled with comprehensive Renasys NPWT foam dressing. Tissue samples from the wound bed and blood were obtained at study onset and after 8 days of treatment; however, a deviation of 1 day more or less was exceptionally allowed. Clinical data were collected at the baseline visit. This study was approved by the Jagiellonian University Bioethics Committee in accordance with the Declaration of Helsinki. Written informed consent was obtained from patients prior to inclusion.

\section{Gene methylation arrays}

DNA was isolated from samples obtained at two time points (pre- and post-treatment). Bisulfite conversion was performed using $500 \mathrm{ng}$ of DNA with EZ DNA Methylation-Direct Kit (Zymo, Irvine, CA, USA). The next steps were performed according to the Illumina protocol of Methylation EPIC kit (Illumina, San Diego, CA, USA). Bisulfite-converted-DNA was amplified (whole-genome amplification), fragmented, and precipitated. After resuspension, DNA was hybridized onto Bead Chips for $24 \mathrm{~h}$ at $37^{\circ} \mathrm{C}$. The non-hybridized and nonspecifically hybridized DNA was washed away following extension https://ec.bioscientifica.com https://doi.org/10.1530/EC-19-0373 (c) 2019 The authors Published by Bioscientifica Ltd
This work is licensed under a Creative Commons Attribution-NonCommercial 4.0 International License. ded from Bioscientifica.com at 04/26/2023 12:13:41PM via free access 
and staining. Imaging was performed using HiScan (Illumina, USA).

\section{Bioinformatics and statistical analysis}

For analysis of the characteristics of the study groups, we used Statistica Software v. 12.0 (StatSoft, Tulsa, OK, USA). The Shapiro-Wilk test was performed to check normality. Differences between groups were assessed by the $t$-test or $U$ test for normally and non-normally distributed continuous variables, respectively, and by chi-square or Fischer's exact test for categorical variables. Continuous variables are presented as mean \pm s.D. or median (interquartile range).

Quality control of the samples was performed using bioconductor package 'minfi' in statistical software $\mathrm{R}$ (version 3.5.0), according to 'The minfi User's Guide'. Using the 'minfi' package a Subset-quantile Within Array Normalization (SWAN) procedure was applied to the raw data.

Target statistical analysis was performed using package 'ChAMP' in statistical software $\mathrm{R}$ version 3.5.0. In total, 72 samples (half pre-treatment and another half post-treatment) and 846,232 features were analyzed. Four different comparisons were performed: (1 and 2) difference between pre- and post-treatment in control arm as well as NPWT arm; (3 and 4) difference between control and NPWT arms pre-treatment as well as or posttreatment. Prior to the endpoint analysis, confounding effects associated with batch (methylation array) and gender were removed. Differentially methylated regions were detected using the 'ProbeLasso' algorithm (13) with FDR 0.05. Gene set enrichment analysis was performed using Fisher's exact test with FDR 0.05. The SEPIRA package was used as implemented in R Bioconductor. The cell counts were estimated as proposed by the authors of EpiDISH and FlowSorted.Blood.EPIC packages (14).

\section{Results}

The clinical characteristics of the study groups are shown in Table 1; of note, borderline differences occurred for BMI and HbA1c level. The NPWT group was, however, characterized by much greater wound area: 16.8 (IQR: 6.2-20.2) vs 1.4 (IQR: $1.0-2.2) \mathrm{cm}^{2}(P=0.0003)$, in line with patients' original treatment allocation.

First, we checked whether samples from the two cohorts differed in methylation status before treatment. Only one region was differentially methylated between these samples at chromosome 5 (5:140753380140753692). This site encodes the protocadherin gamma subfamily, PCDHGA4, a cell adhesion protein (dependent on calcium) which is involved in the maintenance of specific neuronal connections in the brain (15).

Next, we looked into the effects of NPWT and standard therapy after treatment. Fifty-seven differentially methylated genes were found. After being subjected to enrichment analysis, they were mainly located on chromosomes 6 (chr6p21) and 20 (chr20p13). These areas are associated with DNA repair and autocrine signaling via retinoic acid receptor (Table 2 ).

Subsequently, we performed a differential analysis of the two time points - pre- and post-treatment. The analysis revealed 426 differentially methylated regions in the NPWT group (presented in Supplementary Table 1, see section on supplementary data given at the end of this article). A heatmap with regions characterized by the largest differential methylation in the NPWT

Table 1 Baseline characteristics of the study groups.

\begin{tabular}{lc} 
& NPWT \\
\hline Participants, $n$ & 23 \\
Male, $n$ & 19 \\
Age, years & $68.3 \pm 8.5$ \\
BMI, kg/m² & $26.3(24.7-29.7)$ \\
Diabetes duration, years & $14.1 \pm 1.5$ \\
HbA1c, \% & $6.8(6.0-7.6)$ \\
HbA1c, mmol/mol & $50.8(42.1-59.6)$ \\
eGFR, mL/min/1.73 m² (CKD EPI) & $89.0(66.0-92.0)$ \\
Insulin therapy, $n$ & 20 \\
Smoking current/former/never, $n$ & $3 / 11 / 6$ \\
Wound duration, weeks & $12.0(10.1-22.9)$ \\
Wound area, cm ${ }^{2}$ & $16.8(6.2-20.2)$ \\
CRP, mg/L & $6.08(0.95-26.13)$ \\
\hline
\end{tabular}

\begin{tabular}{cccc}
\hline Standard therapy & & $P$ value \\
\cline { 1 - 1 } 13 & & N/A \\
10 & & 0.70 \\
$64.9 \pm 7.1$ & & 0.23 \\
$29.2(27.1-32.7)$ & & 0.06 \\
$17.0 \pm 2.1$ & & 0.27 \\
$7.6(7.1-8.1)$ & & 0.08 \\
$59.6(54.1-65.0)$ & & 0.08 \\
$72.0(52.0-87.0)$ & & 0.25 \\
12 & & 1.00 \\
$2 / 6 / 4$ & & 0.96 \\
$7.1(3.7-14.5)$ & & 0.06 \\
$1.4(1.0-2.2)$ & 0.00 \\
$6.61(2.78-12.02)$ & 0.81 \\
\hline
\end{tabular}

The age and diabetes duration, which fit into the normal distribution, are presented as mean and standard deviation. The other quantitative traits are presented as median and interquartile range.

https://ec.bioscientifica.com

https://doi.org/10.1530/EC-19-0373 (c) 2019 The authors Published by Bioscientifica Ltd

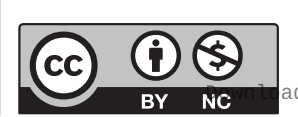

This work is licensed under a Creative Commons Attribution-NonCommercial 4.0 International License. ded from Bioscientifica.com at 04/26/2023 12:13:41PM via free access 
Table 2 List of post treatment enriched processes.

\begin{tabular}{|c|c|c|}
\hline Pathway name & OR $(95 \% \mathrm{Cl})$ & Adj $P$ value \\
\hline chr6p21 & 71.95 & $2.38 \mathrm{E}-38$ \\
\hline chr20p13 & 31.33 & 0.0048 \\
\hline DELACROIX_RAR_BOUND_ES & 9.19 & 0.0050 \\
\hline MORF_DDB̄̄ & 12.8 & 0.0060 \\
\hline
\end{tabular}

Difference between NPWT and control group is presented.

arm is shown in Fig. 1. No such regions were found in the control cohort. Results from the NPWT group were subjected to pathway enrichment analysis in order to find their molecular signatures. It showed 11 processes that were statistically significantly associated with NPWT (Table 3). To test whether the results of differential methylation might be driven by a degree of glycemic control in the study subjects, we performed an additional analysis. Due to limited sample size, it was unfeasible to introduce an interaction term in the linear model. Therefore, to detect an association of CpG sites with HbA1c levels, we performed an analysis of the relationship between patients' $\mathrm{HbA1c}$ and the difference in methylation (matched samples) between pre and post treatment. This analysis showed no sites under the FDR $<0.05$.

Four out of 11 identified processes were associated with complement system activation. Among several genes related to complement system most, apart from one - CLU - which is an inhibitor of complement system activation, were hypermethylated after NPWT leading to inhibition of complement system activation (Table 4).

In order to better understand the mechanism of NPWT action, we performed an analysis of cellular subpopulations on the methylation data of our patients' tissue samples from the wound bed. These samples constituted a cellular mixture, and the analysis was based on molecular markers specific for cellular populations (16). The results of our analysis suggested that NPWT seemed to unload the wound from epithelial cells and fibroblasts, as the number of these cell types decreased more in the treatment arm than in the control arm. Moreover, NPWT led to a decline in most of the inflammatory cells, suggesting that prolonged inflammation was being reduced (Supplementary Table 2). Neutrophils, known for their potency for phagocytosis, were the only subpopulation for which an increase was observed. Additionally, the only inflammatory cell subpopulation which did not decrease in the NPWT arm as much as in the control group was that of the monocytes. Given the well-established phenotypic switch from proinflammatory to anti-inflammatory macrophages that takes place in wound healing, we searched for non-direct results which would support this hypothesis. To further refine our findings, we used the SEPIRA approach (17). In short, we trained a Gene Regulatory Network with GTEx expression data (spleen and blood for reference of expression in immune cells, as well as muscle and skin, for estimation of the target network) and then tested the regulatory activity of the selected transcription factors using the expression (9) and the methylation data. Using the above-described approach together with standard Wilcoxon's signed-rank test, we found transcription factors that were differentially activated pre and post treatment in the NPWT arm. The results showed that the activation of many transcription factors responsible for macrophage activation had changed between the two time points in NPWT arm (Supplementary Table 3).

\section{Discussion}

Here, for the first time in the literature, we provide evidence of the molecular mechanism of NPWT in the

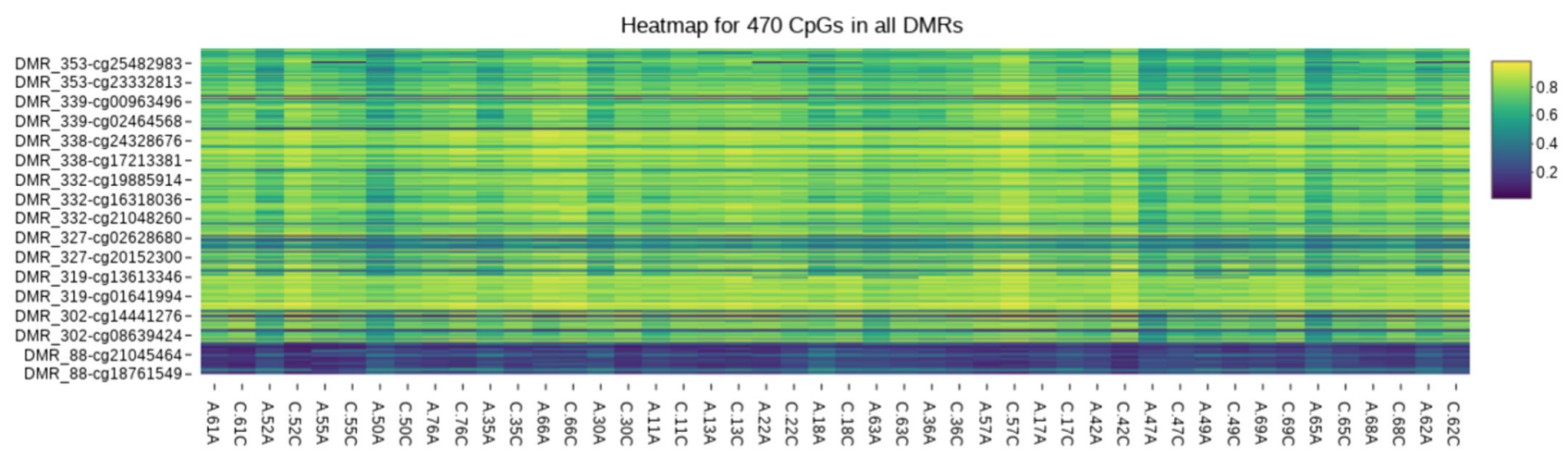

Figure 1

Heatmap presenting the largest differentially methylated regions (all regions contain more than 45 probes with FDR <0.05). Only samples in the NPWT arm are included. Samples are presented in pairs; in each pair, the first sample is pre-treatment and the second is post-treatment.

https://ec.bioscientifica.com

https://doi.org/10.1530/EC-19-0373 (c) 2019 The authors Published by Bioscientifica Ltd

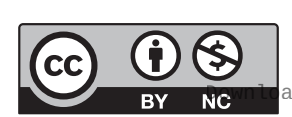

This work is licensed under a Creative Commons Attribution-NonCommercial 4.0 International License. ded from Bioscientifica.com at 04/26/2023 12:13:41PM 
Table 3 List of enriched processes for NPWT group (post-treatment vs pre-treatment).

\begin{tabular}{l}
\hline Pathway name \\
\hline chr6p21 \\
KEGG_ANTIGEN_PROCESSING_AND_PRESENTATION \\
REACTOME_INITIAL_TRIGGERING_OF_COMPLEMENT \\
MODULE_130 \\
KEGG_TYPE_I_DIABETES_MELLITUS \\
MOREAUX_MULTIPLE_MYELOMA_BY_TACI_UP \\
FEVR_CTNNB1_TARGETS_UP \\
BRIDEAU_IMPRINTED_GENES \\
BIOCARTA_COMP_PATHWAY \\
LEI_MYB_TARGETS \\
MIKKELSEN_ES_ICP_WITH_H3K4ME3
\end{tabular}

\begin{tabular}{r}
\hline OR $(95 \% \mathrm{Cl})$ \\
\hline 27.25 \\
9.04 \\
39.70 \\
22.47 \\
13.14 \\
3.54 \\
2.84 \\
9.29 \\
22.68 \\
3.79 \\
2.81
\end{tabular}

\begin{tabular}{l}
\hline Adj $\boldsymbol{P}$ value \\
\hline $4.94 \mathrm{E}-76$ \\
0.0023 \\
0.0024 \\
0.0024 \\
0.0052 \\
0.0052 \\
0.0058 \\
0.0065 \\
0.0068 \\
0.0068 \\
0.0068
\end{tabular}

chronic wound bed of DFUs, namely its impact on DNA methylation of genes in several pathways. In particular, we show that the clinical effect of NPWT may result from an inhibition of complement activation. Among the genes that were hypermethylated and thus inhibited after NPWT, we identified those that coded proteins playing a key role in complement system activation, such as $\mathrm{C} 2, \mathrm{C} 3$, C4A and C4B (18). This is likely to substantially affect the process of activating the complement system. The finding from this study is supported by our previous data on the impact of NPWT on the differential gene expression in DFU patients with T2DM (9). Among genes upregulated after NPWT, we identified C1QBP (Complement component 1 , q subcomponent binding protein), which confirms that the NPWT effects may result from inhibiting the complement system.

Wound healing is an orchestrated process in which several phases occur one after another. The inflammatory stage is followed by proliferative and remodeling phases (19). Poorly healing wounds, such as DFUs, do not follow this scheme and remain predominantly in the chronic inflammatory stage. Healing also hinges on the tissue formation phase, when cells within the wound bed become proliferative and migratory (19). One can hypothesize that the NPWT-induced complement inhibition may allow cells to move onto the next stages of wound healing. Diabetes induces chronic inflammation via prolonged M1 macrophage polarization whose phenotype is similar to 'inflammatory macrophages' which are seen in early stages of wound healing (20). Of note, the complement system is activated in both acute and chronic processes (21). The results of the study performed on $\mathrm{db} / \mathrm{db}$ and control mice has shown that complement activation (checked by C5a concentration) was three times higher in diabetic animals than in healthy controls (22). A similar effect has been observed for C3 fragments (C3a, C3b, iC3b) (22). Furthermore, the utilization of the classical complement pathway inhibitor, PIC1, led not only to a decrease of complement activation by $25 \%$, but also reduced a number of infiltrating neutrophils. It was also shown that complement activation differed between non-diabetic Zucker lean controls and Zucker Diabetic fatty rats following cardiac ischemia and reperfusion and was also higher in the latter group, accompanied by both larger infarct size and neutrophil accumulation (23). In the same model, use of complement inhibitor FUT-175 led to a decrease of complement activation and neutrophil-mediated myocardial cell death (23). Finally, another study performed on C3-deficient mice showed faster progression of wound healing compared to mice with a normally activated complement system (24). The authors also showed that reconstitution of C3-/- mice with serum from $\mathrm{C}+/+$ mice abrogated the healing, which suggests that activation of a complement system might have a negative effect on the progression of wound healing. Similar results were obtained for the

Table 4 Results of methylation levels of genes involved in the complement system pathway at two times points (pre-treatment and post-treatment) in the NPWT group.

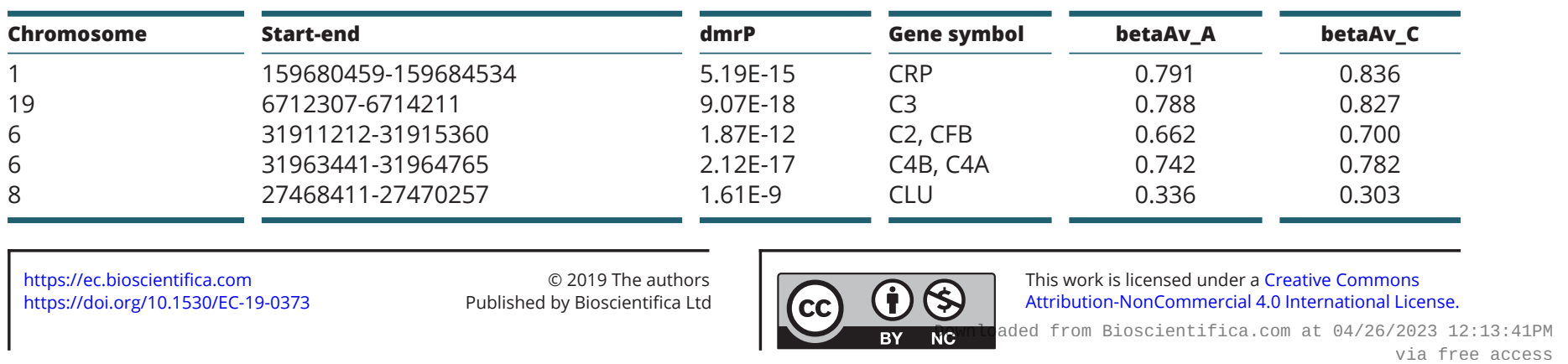


C5 complement element. The inhibition of complement also led to increased vascularization of the wounds, enabling cells to move to the next stage of the healing process. These results have led to a generation of several complement inhibitors, although few have been applied to clinical practice, with the majority undergoing development in the pre-clinical phases of trials (25). Of note, the recent study focusing on the safety of long-term C3 inhibitor compstatin (Cp40 analog) use in the animal model of non-human primates showed that it accelerated wound healing with no weakening of immune system or increased susceptibility to infections compared to the control group (26). Since chronic DFUs may heal better with NPWT, which according to our experiments, may be mediated through complement inhibition, these inhibitors might also prove to be valuable options for DFU treatment. Interestingly, earlier reports described that larval debridement therapy (also known as maggot therapy) might act, among other mechanisms, through the inhibition of the complement system (27). Maggot therapy currently belongs, as does NPTW, to a group of supportive therapies in DFU that are sometimes used to accelerate wound healing.

We also looked into possible cellular subpopulation changes which take place during the NPWT therapy. We observed a removal of most of the inflammatory cells from the wound bed. Only the number of neutrophils increased. Neutrophils are believed to be responsible for complement activation and creation of pro-inflammatory feed-forward loop. At the same time, however, it is known that apart from their effects on the oxidative burst, they play an important role in fine-tuning the inflammatory response as they can express and produce anti-inflammatory cytokines (IL-1a, TGF-B), colony-stimulating factors (G-CSF, GM-CSF) and immunomodulatory cytokines (28). It was also shown that defensin human neutrophil peptide-1 (HNP-1) is able to inhibit activation of the classical complement pathway by inhibition of C1q hemolytic activity (29). The effect of complement inhibition by neutrophils has also been reported. Moreover, since it is known that diabetes itself induces uncontrolled complement system activation, which might lead to vascular events, neuropathy, retinopathy and DFS (30,31, 32), the NPWT therapy might have a more pronounced effect in diabetic patients and differently influence the cellular composition of the wound. Moreover, the only inflammatory cells that did not decease in the NPWT arm as much as in the control group were the monocytes. Possible explanation for the results observed in our research is that monocytes residing in the wound are being transformed into anti-inflammatory macrophages (M2) and advance the healing process in NPWT more than in the control arm. It was shown that neutrophils have the ability to polarize the monocytes to M2 macrophages (33). Our analysis of the methylation state of transcription factors seemed to support this hypothesis as it showed a number of transcription factors earlier proven to be associated with macrophage polarization as well as wound healing. IRF6 was shown to be crucial for macrophage activation (34), enforced expression of Hoxa3 was shown to promote resolution of the inflammation and wound healing in diabetic mice by inhibition of proinflammatory gene expression and induction of anti-inflammatory phenotype (35). A similar effect was seen for NR1D1 (36). Expression of GATA3 had no effect on M1 macrophage markers, but enhanced the M2 phenotype (37). Apart from transcription factors responsible for macrophage polarization, our analysis showed some other transcription factors involved in the wound healing process. For example, Nrf2 was shown to protect the cells from oxidative stress during wound healing (38). Additionally, zinc finger proteins activate Notch1, which is responsible for mesenchymal cell differentiation (39). TRIP6 is known to influence acquisition of locomotor phenotype, its knockdown leads to increased cell motility and might (40). Also, TEAD has been shown to stimulate cell proliferation and migration (41). SOX10 also influences EMT and motility (42). However, it should be emphasized that the hypothesis described earlier is currently not supported by any direct experimental evidence based on reference cell sorting technique. We are only able to provide the results of in silico analysis.

Our results showing NPWT's modification of the wound-healing cascade are in line with a murine model study on epigenetic changes in bone marrow progenitor cells that influence the inflammatory phenotype and alter wound healing in T2DM (43). So far, only a few studies on methylation changes have been conducted on DFUs in humans; however, none of them examined whole-genome methylation changes after NPWT. One study utilizing genome-wide DNA methylation within the context of DFUs was performed involving patientderived fibroblasts. In this study, an epigenetic pattern of metabolic memory in these cells was proven and elicited targets for the functioning of wound healing (44).

The study has several limitations and shortcomings. First, we used a non-random study design, which may have predisposed this research to biases by a number of confounding factors, such as potential differences in baseline characteristics (e.g. wound size). Risk of such

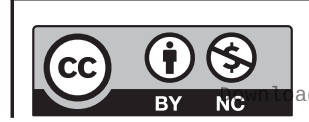


biases in our study should be considered significant, as is usually the case in non-randomized clinical experiments. We decided not to use randomization in patients with larger ulcers due to ethical reasons. DFU is a dangerous, potentially disabling and life-threatening complication, and NPWT has well-documented benefits in these patients (reviewed in (9)). We assigned patients to groups according to local and international research and clinical practice as well as available guidelines concerning on NPWT use in DFU $(10,11,12,45,46)$. It is of note, however, that initially the samples from the two cohorts differed in methylation status before treatment only in one chromosomal region, while many more such differences were detected after treatment. Nevertheless, we cannot exclude that even this single chromosomal region difference may have resulted in a selection bias. The potential impact of hypoglycemic therapies used in our study groups could have also potentially influenced our results. However, it should be noted that almost all patients in this study were on insulin at the onset of study entry. It is thus unlikely that hypoglycemic therapy had an effect on the study results; however, we cannot exclude the possible effect of other known and unknown factors. Thirdly, the number of samples was rather limited, which influenced the power of the analysis. Thus, some regions characterized by smaller methylation differences could have remained undetected. Additionally, the results of this study should be considered in the context of the duration of NPWT use. Longer, as well as shorter experiments, might have provided different results as other molecular mechanisms could exert an effect on healing. The length of NPWT use varies across institutions from a few days to several months (reviewed in (9)); the 8-day duration used in this study reflects our routine clinical practice (12). Of note, in a similar study, NPWT was shown to exert local molecular effects after 7 days of treatment (47). Finally, no other experimental method was used to confirm these findings, including functional experiments or validation in independent cohorts.

\section{Conclusions}

In summary, we found initial evidence that the effect of NPWT on DFUs may be mediated through epigenetic alterations, resulting mainly in the inhibition of complement system activation. This discovery holds potential clinical implications and may be of value in guiding the development of new therapies for DFUs.

\section{Supplementary data}

This is linked to the online version of the paper at https://doi.org/10.1530/ EC-19-0373.

\section{Declaration of interest}

The authors declare that there is no conflict of interest that could be perceived as prejudicing the impartiality of the research reported.

\section{Funding}

The study was funded by the National Science Centre in Poland through the Opus Grant 'Assessment of molecular mechanisms of negative pressure wound therapy in the treatment of neuropathic ulceration in diabetic foot syndrome' to M T M (Nr 2013/11/B/NZ5/03298). The Omicron laboratory was funded by the European Union (7th Framework Programme, Call number FP7-REGPOT-2011-1, Grant number 286038).

\section{Data availability}

The datasets generated during and/or analyzed during the current study are available from the corresponding author on reasonable request.

\section{Author contribution statement}

$A L S, S B, J H, B K W, E P, M T M$ performed the research. $M S, J H$, P K analyzed the data. A L S, S B, M S, B K W, P W, M T M designed the research and interpreted the data. A L S, P W, M T M wrote the paper. All authors contributed to critical revision of the manuscript and approved its publication. M T M and P W are the guarantors of this work.

\section{Acknowledgements}

The authors thank Dr Claudia Frankfurter, University of Toronto, Faculty of Medicine, for linguistic review of the manuscript.

\section{References}

1 Boulton AJ. The diabetic foot: grand overview, epidemiology and pathogenesis. Diabetes/Metabolism Research and Reviews 200824 (Supplement 1) S3-S6. (https://doi.org/10.1002/dmrr.833)

2 Zhang P, Lu J, Jing Y, Tang S, Zhu D \& Bi Y. Global epidemiology of diabetic foot ulceration: a systematic review and meta-analysis. Annals of Medicine 201749 106-116. (https://doi.org/10.1080/07853 890.2016.1231932)

3 Jeffcoate WJ, Vileikyte L, Boyko EJ, Armstrong DG \& Boulton AJM. Current challenges and opportunities in the prevention and management of diabetic foot ulcers. Diabetes Care 201841 645-652. (https://doi.org/10.2337/dc17-1836)

4 Apelqvist J, Willy C, Fagerdahl AM, Fraccalvieri M, Malmsjö M, Piaggesi A, Probst A \& Vowden P. EWMA document: negative pressure wound therapy. Journal of Wound Care 201726 (Supplement 3) S1-S154. (https://doi.org/10.12968/jowc.2017.26.Sup3.S1)

5 Game FL, Apelqvist J, Attinger C, Hartemann A, Hinchliffe RJ, Löndahl M, Price PE, Jeffcoate WJ \& International Working Group on the Diabetic Foot. Effectiveness of interventions to enhance healing of chronic ulcers of the foot in diabetes: a systematic review. Diabetes/Metabolism Research and Reviews 201632 (Supplement 1) 154-168. (https://doi.org/10.1002/dmrr.2707)

6 Borys S, Hohendorff J, Frankfurter C, Kiec-Wilk B \& Malecki MT. Negative pressure wound therapy use in diabetic foot syndrome-from
This work is licensed under a Creative Commons Attribution-NonCommercial 4.0 International License. ded from Bioscientifica.com at 04/26/2023 12:13:41PM 
mechanisms of action to clinical practice. European Journal of Clinical Investigation 201949 e13067. (https://doi.org/10.1111/eci.13067)

7 Shi B, Chen SZ, Zhang P \& Li JQ. Effects of vacuum-assisted closure (VAC) on the expressions of MMP-1, 2, 13 in human granulation wound. Zhonghua Zheng Xing Wai Ke Za Zhi 200319 279-281.

8 Derrick KL, Norbury K, Kieswetter K, Skaf J \& McNulty AK. Comparative analysis of global gene expression profiles between diabetic rat wounds treated with vacuum-assisted closure therapy, moist wound healing or gauze under suction. International Wound Journal 20085 615-624. (https://doi.org/10.1111/j.1742481X.2008.00544.x)

9 Borys S, Ludwig-Slomczynska AH, Seweryn M, Hohendorff J, Koblik T, Machlowska J, Kiec-Wilk B, Wolkow P \& Malecki MT. Negative pressure wound therapy in the treatment of diabetic foot ulcers may be mediated through differential gene expression. Acta Diabetologica 201956 115-120. (https://doi.org/10.1007/s00592-0181223-y)

10 Lavery LA, Boulton AJ, Niezgoda JA \& Sheehan P. A comparison of diabetic foot ulcer outcomes using negative pressure wound therapy versus historical standard of care. International Wound Journal 20074 103-113. (https://doi.org/10.1111/j.1742-481X.2007.00317.x)

11 Blume PA, Walters J, Payne W, Ayala J \& Lantis J. Comparison of negative pressure wound therapy using vacuum-assisted closure with advanced moist wound therapy in the treatment of diabetic foot ulcers: a multicenter randomized controlled trial. Diabetes Care 2008 31 631-636. (https://doi.org/10.2337/dc07-2196)

12 Borys S, Hohendorff J, Koblik T, Witek P, Ludwig-Slomczynska AH, Frankfurter C, Kiec-Wilk B \& Malecki MT. Negative-pressure wound therapy for management of chronic neuropathic noninfected diabetic foot ulcerations - short-term efficacy and long-term outcomes. Endocrine 201862 611-616. (https://doi.org/10.1007/ s12020-018-1707-0)

13 Butcher LM \& Beck S. Probe Lasso: a novel method to rope in differentially methylated regions with $450 \mathrm{~K}$ DNA methylation data. Methods 201572 21-28. (https://doi.org/10.1016/j. ymeth.2014.10.036)

14 Salas LA, Koestler DC. FlowSorted.Blood.EPIC: Illumina EPIC data on immunomagnetic sorted peripheral adult blood cells. R package version 1.3.0. Bioconductor, 2019. (available at:https://bioconductor org/packages/devel/data/experiment/html/FlowSorted.Blood.EPIC. html)

15 Yagi T \& Takeichi M. Cadherin superfamily genes: functions, genomic organization, and neurologic diversity. Genes and Development 200014 1169-1180.

16 Teschendorff AE, Breeze CE, Zheng SC \& Beck S. A comparison of reference-based algorithms for correcting cell-type heterogeneity in Epigenome-Wide Association Studies. BMC Bioinformatics 201718 105. (https://doi.org/10.1186/s12859-017-1511-5

17 Chen Y, Widschwendter M \& Teschendorff AE. Systems-epigenomics inference of transcription factor activity implicates aryl-hydrocarbonreceptor inactivation as a key event in lung cancer development. Genome Biology 201718 236. (https://doi.org/10.1186/s13059-0171366-0)

18 Hajishengallis G, Reis ES, Mastellos DC, Ricklin D \& Lambris JD. Novel mechanisms and functions of complement. Nature Immunology 201718 1288-1298. (https://doi.org/10.1038/ni.3858)

19 Gonzalez AC, Costa TF, Andrade ZA \& Medrado AR. Wound healing a literature review. Anais Brasileiros de Dermatologia 201691 614-620. (https://doi.org/10.1590/abd1806-4841.20164741)

20 Bannon P, Wood S, Restivo T, Campbell L, Hardman MJ \& Mace KA. Diabetes induces stable intrinsic changes to myeloid cells that contribute to chronic inflammation during wound healing in mice. Disease Models and Mechanisms 20136 1434-1447. (https://doi. org/10.1242/dmm.012237)

21 Petersen NE, Elmgreen J, Teisner B \& Svehag SE. Activation of classical pathway complement in chronic inflammation. Elevated levels of circulating C3d and C4d split products in rheumatoid arthritis and Crohn's disease. Acta Medica Scandinavica 1988223 557-560.

22 Cunnion KM, Krishna NK, Pallera HK, Pineros-Fernandez A, Rivera MG, Hair PS, Lassiter BP, Huyck R, Clements MA, Hood AF, et al. Complement activation and STAT4 expression are associated with early inflammation in diabetic wounds. PLOS ONE 201712 e0170500. (https://doi.org/10.1371/journal.pone.0170500)

23 La Bonte LR, Davis-Gorman G, Stahl GL \& McDonagh PF. Complement inhibition reduces injury in the type 2 diabetic heart following ischemia and reperfusion. American Journal of Physiology: Heart and Circulatory Physiology 2008294 H1282-H1290. (https://doi. org/10.1152/ajpheart.00843.2007)

24 Rafail S, Kourtzelis I, Foukas PG, Markiewski MM, DeAngelis RA, Guariento M, Ricklin D, Grice EA \& Lambris JD. Complement deficiency promotes cutaneous wound healing in mice. Journal of Immunology 2015194 1285-1291. (https://doi.org/10.4049/ jimmunol.1402354)

25 Cazander G, Jukema GN \& Nibbering PH. Complement activation and inhibition in wound healing. Clinical and Developmental Immunology 20122012 534291. (https://doi. org/10.1155/2012/534291)

26 Reis ES, Berger N, Wang X, Koutsogiannaki S, Doot RK, Gumas JT, Foukas PG, Resuello RRG, Tuplano JV, Kukis D, et al. Safety profile after prolonged C3 inhibition. Clinical Immunology 2018197 96-106. (https://doi.org/10.1016/j.clim.2018.09.004)

27 Tamura T, Cazander G, Rooijakkers SH, Trouw LA \& Nibbering PH. Excretions/secretions from medicinal larvae (Lucilia sericata) inhibit complement activation by two mechanisms. Wound Repair and Regeneration 201725 41-50. (https://doi.org/10.1111/wrr.12504)

28 Tecchio C, Micheletti A \& Cassatella MA. Neutrophil-derived cytokines: facts beyond expression. Frontiers in Immunology 20145 508. (https://doi.org/10.3389/fimmu.2014.00508)

29 van den Berg RH, Faber-Krol MC, van Wetering S, Hiemstra PS \& Daha MR. Inhibition of activation of the classical pathway of complement by human neutrophil defensins. Blood 199892 3898-3903.

30 Hansen TK, Thiel S, Knudsen ST, Gravholt CH, Christiansen JS, Mogensen CE \& Poulsen PL. Elevated levels of mannan-binding lectin in patients with Type 1 diabetes. Journal of Clinical Endocrinology and Metabolism 200388 4857-4861. (https://doi. org/10.1210/jc.2003-030742)

31 Kaunisto MA, Sjölind L, Sallinen R, Pettersson-Fernholm K, Saraheimo M, Fröjdö S, Forsblom C, Fagerudd J, Hansen TK, Flyvbjerg A, et al. Elevated MBL concentrations are not an indication of association between the MBL2 gene and type 1 diabetes or diabetic nephropathy. Diabetes 200958 1710-1714. (https://doi.org/10.2337/ db08-1495)

32 Guan LZ, Tong Q \& Xu J. Elevated serum levels of mannose-binding lectin and diabetic nephropathy in type 2 diabetes. PLOS ONE 2015 10 e0119699. (https://doi.org/10.1371/journal.pone.0119699)

33 Yang W, Tao Y, Wu Y, Zhao X, Ye W, Zhao D, Fu L, Tian C, Yang J, He F, et al. Neutrophils promote the development of reparative macrophages mediated by ROS to orchestrate liver repair. Nature Communications 201910 1076. (https://doi.org/10.1038/s41467-01909046-8)

34 Li C, Ying W, Huang Z, Brehm T, Morin A, Vella AT \& Zhou B. IRF6 regulates alternative activation by suppressing PPAR $\gamma$ in male murine macrophages. Endocrinology 2017158 2837-2847. (https://doi. org/10.1210/en.2017-00053)

35 Al Sadoun H, Burgess M, Hentges KE \& Mace KA. Enforced expression of Hoxa3 inhibits classical and promotes alternative activation of macrophages in vitro and in vivo. Journal of Immunology $2016 \mathbf{1 9 7}$ 872-884. (https://doi.org/10.4049/jimmunol.1501944)

36 Ma H, Zhong W, Jiang Y, Fontaine C, Li S, Fu J, Olkkonen VM, Staels B \& Yan D. Increased atherosclerotic lesions in LDL receptor 
deficient mice with hematopoietic nuclear receptor Rev-erb $\alpha$ knockdown. Journal of the American Heart Association 20132 e000235.

37 Wang T, Feldman AL, Wada DA, Lu Y, Polk A, Briski R, Ristow K, Habermann TM, Thomas D, Ziesmer SC, et al. GATA-3 expression identifies a high-risk subset of PTCL, NOS with distinct molecular and clinical features. Blood 2014123 3007-3015. (https://doi. org/10.1182/blood-2013-12-544809)

38 Braun S, Hanselmann C, Gassmann MG, auf dem Keller U, BornBerclaz C, Chan K, Kan YW \& Werner S. Nrf2 transcription factor, a novel target of keratinocyte growth factor action which regulates gene expression and inflammation in the healing skin wound. Molecular and Cellular Biology 200222 5492-5505. (https://doi. org/10.1128/mcb.22.15.5492-5505.2002)

39 Wang C, Liu X, Liu Y, Zhang Q, Yao Z, Huang B, Zhang P, Li N \& Cao $\mathrm{X}$. Zinc finger protein 64 promotes toll-like receptor-triggered proinflammatory and type I interferon production in macrophages by enhancing p65 subunit activation. Journal of Biological Chemistry 2013288 24600-24608. (https://doi.org/10.1074/jbc.M113.473397)

40 Guryanova OA, Sablina AA, Chumakov PM \& Frolova EI. Downregulation of TRIP6 gene expression induces actin cytoskeleton rearrangements in human carcinoma cell lines. Molekulyarnaya Biologiya 200539 905-909. (https://doi.org/10.1007/s11008-0050095-8)

41 Zhang H, Liu CY, Zha ZY, Zhao B, Yao J, Zhao S, Xiong Y, Lei QY $\&$ Guan KL. TEAD transcription factors mediate the function of TAZ in cell growth and epithelial-mesenchymal transition. Journal of Biological Chemistry 2009284 13355-13362. (https://doi. org/10.1074/jbc.M900843200)

42 Tong X, Li L, Li X, Heng L, Zhong L, Su X, Rong R, Hu S, Liu W, Jia B, et al. SOX10, a novel HMG-box-containing tumor suppressor, inhibits growth and metastasis of digestive cancers by suppressing the Wnt/ $\beta$-catenin pathway. Oncotarget 20145 10571-10583. (https://doi.org/10.18632/oncotarget.2512)

43 Gallagher KA, Joshi A, Carson WF, Schaller M, Allen R, Mukerjee S, Kittan N, Feldman EL, Henke PK, Hogaboam C, et al. Epigenetic changes in bone marrow progenitor cells influence the inflammatory phenotype and alter wound healing in type 2 diabetes. Diabetes 2015 64 1420-1430. (https://doi.org/10.2337/db14-0872)

44 Park LK, Maione AG, Smith A, Gerami-Naini B, Iyer LK, Mooney DJ, Veves A \& Garlick JA. Genome-wide DNA methylation analysis identifies a metabolic memory profile in patient-derived diabetic foot ulcer fibroblasts. Epigenetics 20149 1339-1349. (https://doi.org/10.41 61/15592294.2014.967584)

45 Apelqvist J, Willy C, Fagerdahl AM, Fraccalvieri M, Malmsjö M, Piaggesi A, Probst A \& Vowden P. EWMA document: negative pressure wound therapy. Journal of Wound Care 201726 (Supplement3) S1-S154. (https://doi.org/10.12968/jowc.2017.26. Sup3.S1)

46 Game FL, Attinger C, Hartemann A, Hinchliffe RJ, Löndahl M, Price PE, Jeffcoate WJ \& International Working Group on the Diabetic Foot. IWGDF guidance on use of interventions to enhance the healing of chronic ulcers of the foot in diabetes. Diabetes/ Metabolism Research and Reviews 201632 (Supplement 1) 75-83. (https://doi.org/10.1002/dmrr.2700)

47 Wang T, He R, Zhao J, Mei JC, Shao MZ, Pan Y, Zhang J, Wu HS, Yu M, Yan WC, et al. Negative pressure wound therapy inhibits inflammation and upregulates activating transcription factor-3 and downregulates nuclear factor-кB in diabetic patients with foot ulcerations. Diabetes/Metabolism Research and Reviews 201733 e2871. (https://doi.org/10.1002/dmrr.2871)

Received in final form 3 October 2019

Accepted 21 October 2019

Accepted Preprint published online 21 October 2019
This work is licensed under a Creative Commons Attribution-NonCommercial 4.0 International License. ded from Bioscientifica.com at 04/26/2023 12:13:41PM 